\title{
Application of a Personalized 3D-Printed Guide Plate for Implantation of Thoracolumbar Pedicle Screws for Patients with Ankylosing Spondylitis
}

\author{
YI FAN. YU1,2, YAN. XU², QING QING. YAO² AND LI MING. WANG* \\ ${ }^{1}$ Department of Orthopaedic Surgery, Nanjing First Hospital, Nanjing Medical University, Nanjing-210006, ${ }^{2}$ Department of \\ Orthopaedic Surgery, The Affiliated Huaian No.1 People's Hospital of Nanjing Medical University, Huaian-223300, China
}

Yui et al.: Application of Personalized 3D-Printed Guide Plate

To investigate the application of a "personalized" guide plate for pedicle screw insertion in the thoracolumbar spine of patients with ankylosing spondylitis using three-dimensional printing. Twenty ankylosing spondylitis patients with a thoracolumbar vertebral fracture were examined using computed tomography. Anatomic digital imaging and communications in medicine data was reconstructed to stereolithography data through M3D software. Three-dimensional printing was done using fused deposition modeling. The length and diameter of pedicle screw, angle between the pedicle screws and the horizontal plane, angle between the PS and the sagittal plane, and distance between the entry position and midline were measured based on three-dimensional printing. Time of operation, intraoperative blood loss, postoperative drainage volume, time of nailing, time of radiation exposure and Japanese orthopedic association scores were also recorded. No significant difference between the preoperative predicted value and actual values of the pedicle screw was found in the three-dimensional printing group for length and diameter of pedicle screw, angle between the pedicle screw and the horizontal plane, angle between the pedicle screw and the sagittal plane and distance between the entry position and midline. The Time of operation, intraoperative blood loss and postoperative drainage volume of the three-dimensional printing group were significantly better than those of the non- three-dimensional printing group. Moreover, the time of nailing and time of radiation exposure of patients in the three-dimensional printing group were significantly shorter than those of the non-three-dimensional printing group. Japanese orthopedic association scores at 1, 2 and $4 \mathrm{w}$ after surgery in the three-dimensional printing group were significantly higher than those in the non-threedimensional printing group, but there was no significant difference between groups at 6 mo. A personalized guide plate constructed by three-dimensional printing could assist thoracolumbar pedicle screw insertion in ankylosing spondylitis patients.

Key words: Thoracolumbar Pedicle Screws, three dimensional printed guide plate, tomography, digital three dimensional models, surgical procedure

Ankylosing spondylitis (AS) is a chronic inflammatory disease involving predominantly the axial skeleton. Chronic spine changes in AS, including syndesmophyte formation, ankylosis, and osteoporosis, predispose these individuals to spine fractures even after minor trauma ${ }^{[1,2]}$. The reported prevalence of vertebral fracture in AS patients ranges from $0.4 \%$ to $32.4 \%{ }^{[3-}$ 5]. Fractures in the ankylosed thoracolumbar spine are particularly more unstable than those in a healthy spine $^{[6]}$. Non-surgical intervention has often leaded to poor healing of the fracture, resulting in pseudoarthrosis that can make subsequent surgical treatment more difficult ${ }^{[7]}$. Caron et al. ${ }^{[8]}$ retrospectively compared a

*Address for correspondence

E-mail: shen1700477336369@163.com

September-October 2020

Indian Journal of Pharmaceutical Sciences large consecutive series of patients of spine fractures in AS spine. 75 patients received surgical repair and 37 patients received conservative management. Mortality was lower for surgical patients (23\% vs. $51 \%$ ). Westerveld et al. ${ }^{[9]}$ revealed that surgical repair was better for AS patients. Lu et al. ${ }^{[10]}$ demonstrated that conservative treatment resulted mostly in

This is an open access article distributed under the terms of the Creative Commons Attribution-NonCommercial-ShareAlike 3.0 License, which allows others to remix, tweak, and build upon the work non-commercially, as long as the author is credited and the new creations are licensed under the identical terms

Accepted 15 September 2020

Revised 09 September 2020

Received 17 June 2020

Indian J Pharm Sci 2020;82(5):902-908 
pseudarthrosis and progressive neurologic deficit while surgical repair could achieve better fusion and improve neurologic symptoms in 25 AS patients with thoracolumbar fractures.

In recent years, there have been rapid advances in application of "digital medicine" and three-dimensional (3D) printing technology in orthopedic surgery. Using $3 \mathrm{D}$ printing can enable accurate re-construction of a complex anatomic structure for better preoperative planning ${ }^{[11,12]}$. Here, we describe clinical application of a 3D-printed-assisted posterior-segment pedicle screw (PS) system for the treatment of thoracolumbar vertebral fractures in patients with AS.

\section{MATERIALS AND METHODS}

\section{Patient grouping:}

Between 1 January 2015 and 31 December 2017, 42 patients (18 men and 24 women), mean age 58.7 (range, 52-66) with a thoracolumbar vertebral fracture with AS were treated at the Nanjing First Hospital of Nanjing Medical University (Nanjing, China). Fractures occurred at T12 (22 cases) or L1 (20 cases). 20 patients were treated in the $3 \mathrm{D}$ printing group (3DP group), and 22 patients in the non-3D printing group (non-3DP group).

\section{Reconstruction of a digital 3D model and 3D printing:}

Anatomic data obtained by computed tomography (CT; $1.00 \mathrm{~mm}$ of volume thickness, matrix of $512 \times 512$; Sensation, Siemens, Munich, Germany) were transferred to a planning workstation (M3D; MedGraphics, Shanghai, China) using a Digital Imaging and Communications in Medicine interface. A 3D model of thoracolumbar vertebrae was reconstructed on screen. The software allowed the surgeon to simulate PS placement preoperatively. The position direction of entry of PSs was determined on horizontal, sagittal and coronal CT slices of the vertebral body (fig. 1-3), respectively. After the correct position of PSs had been determined, a guide plate (GP) was manufactured to navigate the PSs into the vertebral body (fig. 4). Information on the GP model was stored as a stereolithography file and sent to a $3 \mathrm{D}$ printer. The printing procedure was repeated layer-by-layer until a complete 3D model had been generated. Simulated placement of PSs was also conducted on the model preoperatively.

\section{Surgical procedure:}

Patients were placed prone on a padded spinal frame or operating table with chest rolls. After skin sterilization and draping, a midline skin incision centered over the spinous process at the level of the injury was made. Then, posterior elements were exposed at two levels above and two levels below the level of injury. The latter was confirmed with radiographs. Dissection was continued with electrocautery to the fascia, and widened to the tips of the transverse processes in the thoracic and lumbar spine.

In the 3D printing group (3DP group), thoracic and lumbar PSs were placed into vertebral pedicles by the GP manufactured preoperatively (fig. 5). In the
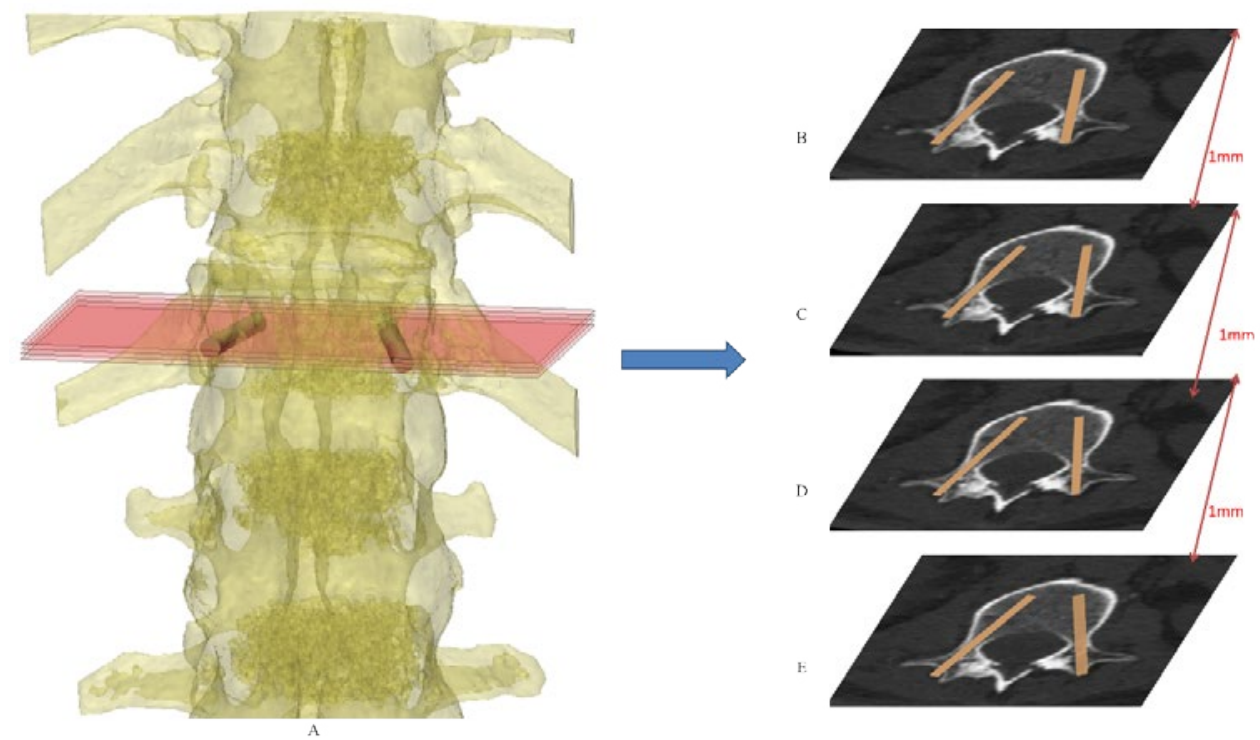

Fig. 1: How the pedicle screw (PS) position was measured on horizontal CT slices, each of which were 1-mm apart (B-E), after the PS had been replaced in the vertebral body by software (A) 


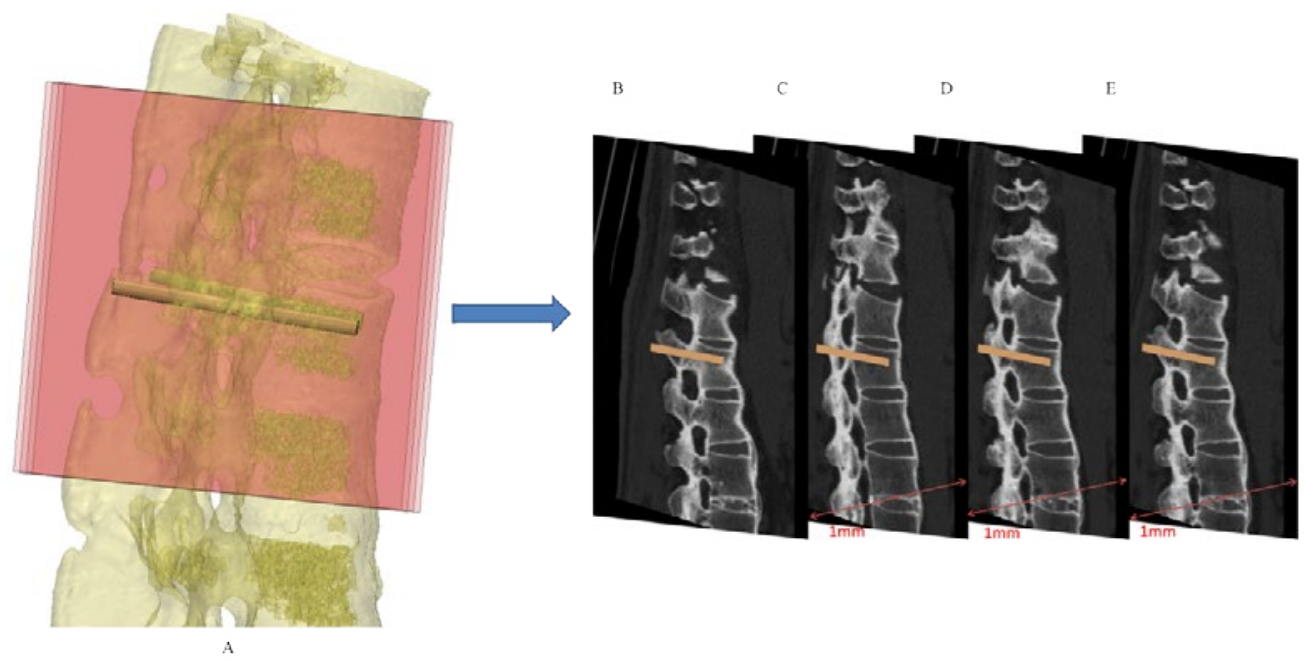

Fig. 2: How the PS position was measured on sagittal CT slices, each of which were 1-mm apart (B-E), after the PS was replaced in the vertebral body by software (A)
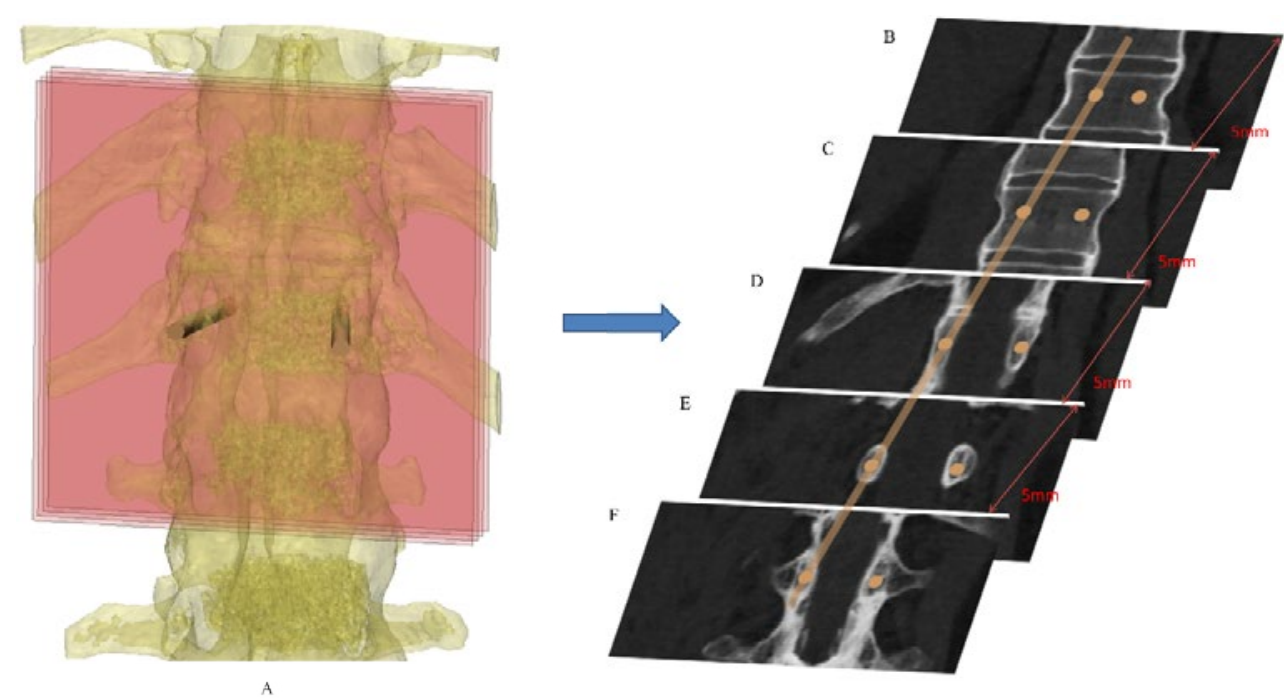

Fig. 3: How the PS position was measured on coronal CT slices, each of which was 5-mm apart (B-F), after the PS was replaced in the vertebral body by software (A)

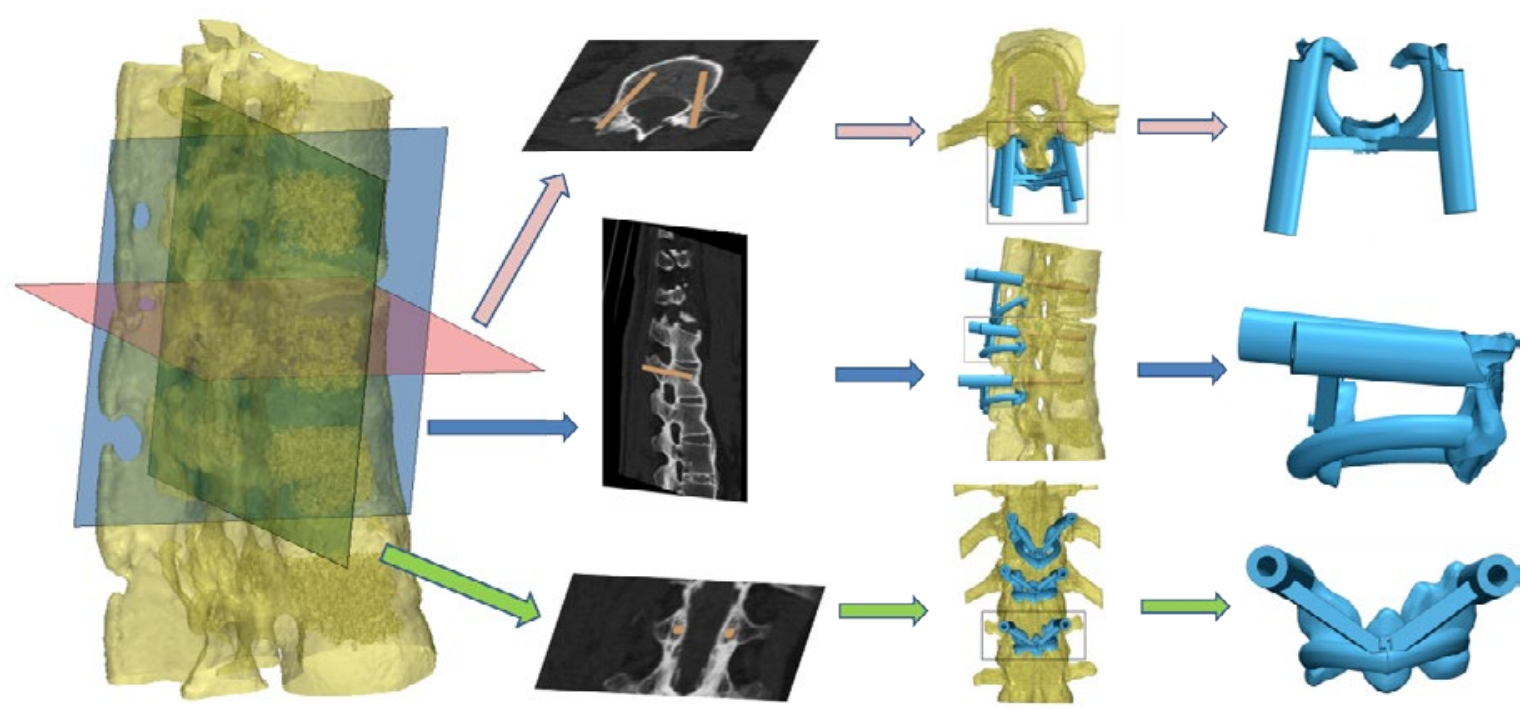

Fig. 4: Guide plate (GP) design. After determining the correct position of the PS on horizontal, sagittal and coronal CT slices, respectively, the GP was manufactured by the software 
non-3D printing group (non-3DP group), thoracic PSs were placed into vertebral pedicles by the Roy-Camille technique, and lumbar PSs were placed using the herringbone crest vertex method. Four segments of the vertebral body were fixed in all cases. Spinal columns were imaged to verify PS position after PS placement. Finally, the rod and crosslinks were placed. Morselized cancellous bone grafts were also harvested from the iliac crest if additional bones were needed. Neuro monitoring was used intra operatively. Finally, the incision was closed.

\section{Postoperative management:}

Postoperatively, a radiograph was obtained to verify PS position. All patients received antibiotic prophylaxis for $48 \mathrm{~h}$. Patients were allowed bed rest for $4 \mathrm{w}$, and then allowed to ambulate with thoracolumbar braces for at least 3 mo depending on the follow-up radiographic evidence of fracture healing.

\section{Outcome measures:}

The theoretical parameters of PSs measured based on the re-constructed 3D model were the: length of PSs (LPS); diameter of PSs (DPS); angle between the PS and the horizontal plane (APSHP); angle between the PS and the sagittal plane (APSSP); distance between the entry position and midline (DEPM) (fig. 6). These parameters were compared with the actual values during surgery (Table 1). The time of operation (TO), intraoperative blood loss (IBL), postoperative drainage volume (PDV), time of nailing (TN), time of radiation exposure (TRE) and Japanese Orthopedic Association
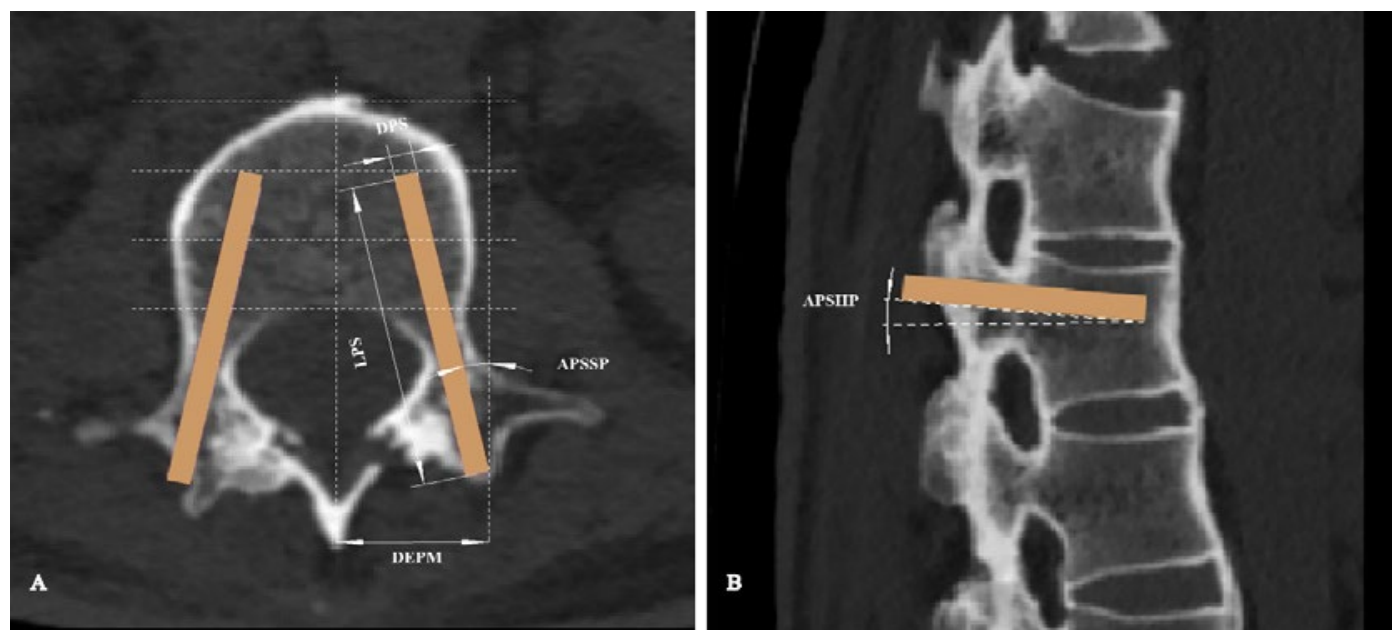

Fig. 5: Measurement of PS parameters. The parameters of PS were measured according to the 3DP model. These were the: length of PSs (LPS), diameter of PSs (DPS), the angle between the PS and the sagittal plane (APSSP), distance between the entry position and midline (DEPM) (A), and the angle between the PS and the horizontal plane (APSHP) (B)
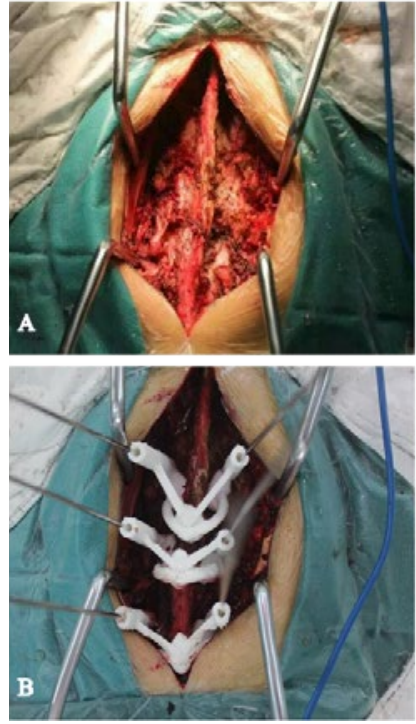
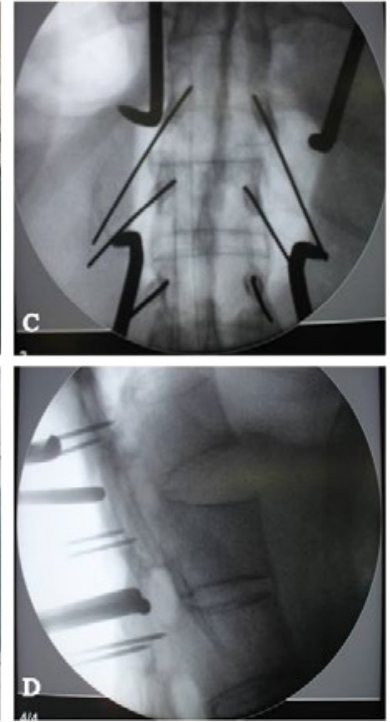
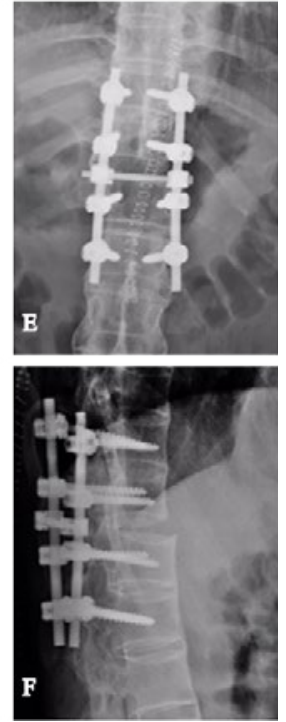

Fig. 6: Surgical procedure. In the 3DP group, thoracic and lumbar PSs were placed into vertebral pedicles with the aid of the GP (A-D). Three days after surgery, radiography showed satisfactory reduction of fractures with correct positioning of the internal fixation (E-F) 
(JOA) scores after surgery were recorded and compared with the corresponding values in patients undergoing the conventional (non-3D printing) procedure at our hospital (Table 2 and 3). Patients were reviewed clinically and imaged 3 days after surgery and followed up for 6 mo.

\section{Statistical analyses:}

Data are the mean \pm standard deviation. The Student's t-test was used to compare data. Comparison of variables between groups was done using the independent sample t-test. $\mathrm{p}<0.5$ was considered significant. SPSS v13.0 (IBM, Armonk, NY, USA) was used for statistical analyses.

\section{RESULTS AND DISCUSSION}

For the predictive parameter values of PSs (LPS, DPS, APSHP, APSSP, DEPM) before surgery, there was no significant difference compared with the actual values intraoperatively $(\mathrm{p}>0.05)$ (Table 1$)$.

The TO, IBL and PDV of the 3DP group were better than those of the non-3DP group $(\mathrm{p}<0.05)$. The TN and TRE of patients in the 3DP group were shorter than those in the non-3DP group $(\mathrm{p}<0.05)$ (Table 2$)$. The JOA scores in the 3DP group 1, 2, and $4 \mathrm{w}$ after surgery were superior to those in the non-3DP group $(\mathrm{p}<0.05)$. However, there was no significant difference after 6 mo $(\mathrm{p}>0.05)$ (Table 3).

The overall prevalence of AS has been estimated to be between $0.1 \%$ and $1.4 \%{ }^{[13]}$. AS changes the biomechanical properties of the spine ${ }^{[14]}$ through ligamentous ossifications, vertebral joints fusion, osteoporosis and kyphosis. These changes can cause spine more susceptible to fractures. These fractures occur in areas of stress, such as thoracolumbar vertebrae $^{[15]}$. Surgical management has become better treatment of these fractures. $\mathrm{Hu}$ and colleagues ${ }^{[16]}$ demonstrated that a posterior-segment PS system for treatment of AS patients with thoracolumbar fractures can elicit favorable clinical results with strong internal fixation and fracture healing. However, finding the position and direction of PSs intra operatively is difficult due to spinal fusion and facet-joint deformity.

At present, the commonly used manual nailing method (e.g., herringbone nail insertion, transverse process) is based on the posterior anatomy of the vertebral body to find the bony mark, and then determination of the entry position. However, the bony marks can be ambiguous due to extensive ossification of the lamina, facet joints and surrounding tissues in patients with AS. Moreover, it is difficult to find the insertion position of PSs intra operatively, which increases the difficulty of surgery considerably. The accuracy of manual insertion of PSs is not high, and penetration of PSs is easily done. Intra operatively, it is often necessary to fully reveal the lateral edge of the lamina and root of the transverse process, and the joint protrusion is "bitten off" part of the bone to find the remaining small joint space, which increases surgical trauma. Intraoperative $\mathrm{C}$-arm fluoroscopy is required to ascertain if PSs have been selected correctly, which increases the radiation exposure to the patient and surgeon.

Development of 3D printing technology has allowed surgeons (after observation of the anatomic structure of fractures preoperatively) to plan how to place PSs faster and more accurately. Lu et al. used 3D technology to navigate $22 \mathrm{PSs}$ in six patients ${ }^{[17]}$. Outcomes showed no

\begin{tabular}{|c|c|c|}
\hline & $\begin{array}{c}\text { Theoretical } \\
\text { value }\end{array}$ & $\begin{array}{l}\text { Actual } \\
\text { value }\end{array}$ \\
\hline $\begin{array}{l}\text { Diameter of pedicle screws } \\
(\mathrm{mm})\end{array}$ & $6.32 \pm 0.24$ & $6.28 \pm 0.25$ \\
\hline Length of pedicle screws $(\mathrm{mm})$ & $43.25 \pm 2.39$ & $42.75 \pm 2.49$ \\
\hline $\begin{array}{l}\text { Angle between pedicle screws } \\
\text { and the horizontal plane }\left({ }^{\circ}\right)\end{array}$ & $5.79 \pm 0.15$ & $5.80 \pm 0.16$ \\
\hline $\begin{array}{l}\text { Angle between pedicle screws } \\
\text { and the sagittal plane }\left({ }^{\circ}\right)\end{array}$ & $11.42 \pm 0.16$ & $11.40 \pm 0.18$ \\
\hline $\begin{array}{l}\text { Distance between the entry } \\
\text { position and midline }(\mathrm{mm})\end{array}$ & $26.16 \pm 0.25$ & $26.08 \pm 0.22$ \\
\hline
\end{tabular}

\begin{tabular}{|c|c|c|}
\hline \multicolumn{2}{|c|}{$\begin{array}{l}\text { TABLE } 2: \text { COMPARISON OP } \\
\text { CONDITIONS BETWEEN THE 3DP } \\
\text { NON-3DP GROUP }(p<0.05 \text { FOR ALL) }\end{array}$} & $\begin{array}{r}\text { SURGICAL } \\
\text { GROUP AND }\end{array}$ \\
\hline & $\begin{array}{c}\text { 3DP group } \\
(n=20)\end{array}$ & $\begin{array}{c}\text { Non-3DP group } \\
(n=22)\end{array}$ \\
\hline Time of operation (min) & $131.25 \pm 7.56$ & $152.04 \pm 5.57$ \\
\hline $\begin{array}{l}\text { Intraoperative blood loss } \\
(\mathrm{mL})\end{array}$ & $224.50 \pm 14.99$ & $306.82 \pm 17.68$ \\
\hline $\begin{array}{l}\text { Postoperative drainage } \\
\text { volume }(\mathrm{mL})\end{array}$ & $249.50 \pm 19.61$ & $330.90 \pm 32.88$ \\
\hline Time of nailing (min) & $6.02 \pm 0.34$ & $7.12 \pm 0.53$ \\
\hline $\begin{array}{l}\text { Time of radiation } \\
\text { exposure (min) }\end{array}$ & $2.16 \pm 0.38$ & $3.10 \pm 0.30$ \\
\hline
\end{tabular}

TABLE 3: JOA SCORES AT POSTOPERATIVE FOLLOW-UP IN THE 3DP GROUP AND NON-3DP GROUP ( $p<0.05$ FOR ALL)

\begin{tabular}{lcc}
\hline JOA score & $\begin{array}{c}\text { 3DP group } \\
(\mathbf{n}=20)\end{array}$ & $\begin{array}{c}\text { Non-3DP group } \\
(\mathbf{n}=\mathbf{2 2})\end{array}$ \\
\hline 1 $\mathrm{W}$ after surgery & $20.25 \pm 1.44$ & $18.45 \pm 1.67$ \\
W after surgery & $20.65 \pm 1.28$ & $18.54 \pm 1.59$ \\
$4 \mathrm{w}$ after surgery & $21.45 \pm 1.24$ & $19.05 \pm 1.55$ \\
6 mo after surgery & $22.55 \pm 1.12$ & $22.23 \pm 1.50$ \\
\hline
\end{tabular}


dislocation on $\mathrm{CT}$, significantly shortened the operation time, and reduced radiation exposure. Mere et al. used guided navigation and a manual method, respectively, to place 54 PSs in 20 patients $^{[18]}$. The prevalence of perforation and migration of PSs were decreased significantly in the navigation group. Zhang et al. completed seven cases of complicated lumbar surgery using guided navigation ${ }^{[19]}$. Their results showed no complications of the spinal cord, nerves, or vascular injury in any patient. Also, intraoperative findings were exactly the same as the preoperative $3 \mathrm{D}$ reconstruction model and measurement results. Postoperative radiography and $\mathrm{CT}$ showed good positioning of PSs.

In the present study, 64-slice spiral CT of the thoracolumbar spine was undertaken before surgery to obtain anatomic data. Combined with computerbased analyses, the appropriate position and direction of entry of PSs were selected on the three levels of the vertebral body (coronal, sagittal and horizontal). After determination of the correct position of PSs, a "personalized" GP for PSs was fabricated layer-bylayer by the fused deposition technique. PS fixation was assisted by intraoperative use of a 3D-printed GP. Results showed no significant difference between preoperative theoretical values and actual intraoperative values for LPS, DPS, APSHP, APSSP or DEPM, suggesting that the personalized GP made with 3D printing technology had high precision. Selection of the insertion position and direction of PSs were accurate, and a good surgical effect was obtained.

Compared with computer-navigation technology, the personalized 3D-printed GP was designed for positioning the guide hole for a single vertebral body. Unlike conventional computer navigation, the GP design did not exceed the interval of a single vertebral body, and the position was not changed intraoperatively or affected by adjacent vertebral bodies. Hence, the accuracy of PS positioning by conventional computer navigation would be affected accordingly. Unlike computer-navigation technology, the prepared GP does not need to be registered, which avoids the change of accuracy caused by manual registration. Only anterior and lateral radiation is needed after PS insertion, which greatly reduces radiation exposure for medical staff and patients during surgery.

The personalized 3D-printed GP was designed for singledirection holes in vertebral bodies, including articular processes, lamina and partial spinous processes. It had an effective matching area with vertebral bone and high accuracy of PS insertion. Simultaneously, GPapplication provided greater flexibility, and its attachment was not tight because of the unevenness of some lamina due to bone hyperplasia or osteophyte formation. We believe that 3D-printed GPs can be applied for patients with structural changes/deformity in bone or with difficult anatomic landmarks. Through the design of a personalized GP, PSs can be inserted accurately and rapidly, thereby reducing the risk of injury to blood vessels, nerves, and cervical spine. Simultaneously, the surgical procedure is uncomplicated, the operation time is short, and excessive exposure to radiation can be avoided.

The present study revealed three main limitations of our system for treatment of patients with a thoracolumbar vertebral fracture with AS. First, this technology can help indicate the anatomic structure of a vertebral body, select the size of PSs, and design the GP for PS implantation. However, 3D printing cannot produce an accurate GP for patients with a severe spinal deformity and anatomic abnormality with metallic artifacts of CT, which rely mainly on the experience of the surgeon and intraoperative $\mathrm{C}$-arm fluoroscopy. Second, the acquisition and processing of data for $3 \mathrm{D}$ printing takes considerable time, which may limit its use for emergency surgery. If the surgical procedure is changed, the design cannot be used. Third, the need for specialized computer software and hardware as well as specially trained personnel would increase the cost of this system. Nevertheless, these problems could be solved as 3D-printing technology develops.

Use of a 3D-printing methods for PS insertion for treatment of thoracolumbar vertebral fractures in patients with AS enabled reasonable preoperative planning and better intraoperative orientation of atrisk structures. This strategy improved the efficacy and accuracy of the surgical procedure and outcomes.

\section{Acknowledgements:}

This study was supported by the National Natural Science Foundation of China (81601612, 81771985) and Key Medical Subjects of Jiangsu Province (BE 2015613, BE 2016763).

\section{Conflict of interests:}

The authors declared no conflict of interest.

\section{REFERENCES}

1. Reinhold M, Knop C, Kneitz C, Disch A. Spine Fractures in Ankylosing Diseases: Recommendations of the Spine Section of the German Society for Orthopaedics and Trauma (DGOU). Global Spine J 2018;8(2):56S-68S. 
2. Vazan M, Ryang YM, Barz M, Török E, Gempt J, Meyer B. Ankylosing Spinal Disease-Diagnosis and Treatment of Spine Fractures. World Neurosurg 2019;123:e162-70.

3. Westerveld L, Verlaan JJ, Oner FC. Spinal fractures in patients with ankylosing spinal disorders: a systematic review of the literature on treatment, neurological status and complications. Eur Spine J 2009;18(2):145-56.

4. Lu ML, Tsai TT, Lai PL, Fu TS, Niu CC, Chen LH, et al. A retrospective study of treating thoracolumbar spine fractures in ankylosing spondylitis. Eur J Orthop Surg Traumatol 2014;24:117-23.

5. Xu J, Li D, Ma RF, Barden B, Ding Y. Application of Rapid Prototyping Pelvic Model for Patients with DDH to Facilitate Arthroplasty Planning: A Pilot Study. J Arthroplasty 2015;30(11):1963-70.

6. Rong X, Wang B, Chen H, Ding C, Deng Y, Ma L, et al. Use of rapid prototyping drill template for the expansive open door laminoplasty: a cadaveric study. Clin Neurol Neurosurg 2016;150:13-7.

7. Braun J, Bollow M, Remlinger G, Eggens U, Rudwaleit M, Distler A, et al. Prevalence of spondylarthropathies in HLAB27 positive and negative blood donors. Arthritis Rheum 1998;41(1):58-67.

8. Cornefjord M, Alemany M, Olerud C. Posterior fixation of subaxial cervical spine fractures in patients with ankylosin६ spondylitis. Eur Spine J 2005;14(4):401-8.

9. Chaudhary SB, Hullinger H, Vives MJ. Management of acut $\epsilon$ spinal fractures in ankylosing spondylitis. ISRN Rheumato. 2011;2011:150484.

10. Fox MW, Onofrio BM, Kilgore JE. Neurological complications of ankylosing spondylitis. J Neurosurg 1993;78(6):871-8.

11. Stenhouse G, Ulbricht C, Khanna M. Spinal injury ir ankylosing spondylitis. Bmj 2014;32(26):348.

12. Westerveld L, Verlaan J, Oner F. Spinal fractures in patients with ankylosing spinal disorders: a systematic review of the literature on treatment, neurological status and complications. Eur Spine J 2009;18(2):145-56.

13. Rampersaud YR, Pik JH, Salonen D, Farooq S. Clinical accuracy of fluoroscopic computer-assisted pedicle screw fixation: a CT analysis. Spine. 2005;30(7):E183-90.

14. Amiot P, Lang K, Putzier M. Comparative results between conventional and computer -assisted pedicle screw installation in the thoracic, lumbar, and sacral spine. Spine 2000;25:60614.

15. Rajasekaran S, Vidyadhara S, Ramesh P. Randomized clinical study to compare the accuracy of navigated and nonnavigated thoracic pedicle screws in deformity correction surgeries. Spine 2007;32(2):56-64.

16. $\mathrm{Hu} \mathrm{Y}$, Yuan ZS, Spiker WR. Deviation analysis of $\mathrm{C} 2$ translaminar screw placement assisted by a novel rapid prototyping drill template: a cadaveric study. Eur Spine J 2013;22(12):2770-76.

17. Knez D, Nahle IS, Vrtovec T, Parent S, Kadoury S. Computerassisted pedicle screw trajectory planning using CT-inferred bone density: A demonstration against surgical outcomes. Med Phys 2019;46:3543-54.

18. Zhang M, Li J, Fang T, Zhao J, Pan W, Wang X, et al. Evaluation of a Three-Dimensional Printed Guide and a Polyoxymethylene Thermoplastic Regulator for Percutaneous Pedicle Screw Fixation in Patients with Thoracolumbar Fracture. Med Sci Monit 2020;14:e920578.

19. Zhao J, Yang L, Zheng S, Qu Y, Zhang X, Kang M, et al. Anovel screw view model of 3D navigation for upper cervical pedicle screw placement: A case report. Medicine 2019;98:e15291. 